\title{
Development of Multipurpose Aerogel Cherenkov Counter
}

\author{
H. Ito*, S. lijima, S. Han, H. Kawai, S. Kodama, D. Kumogoshi, K. Mase, M. Tabata \\ Department of Physics, Graduate School of Science Chiba University, Chiba, Chiba, Japan \\ E-mail: hiroshidhepburn.s.chiba-u.ac.ip
}

\begin{abstract}
We have been developing a multipurpose aerogel Cherenkov counter which enables to identify charged particles at any environment such as a limited space and at high magnetic field. The device is composed of an aerogel radiator, a light guide made of 4 kinds of wavelength shifting (WLS) fibers and a small photo-device (PMT or SiPM). Cherenkov light emitted from the aerogel radiator is collected by the fiber light guide, and transferred to the both ends of the fiber by a total reflection condition. Therefore, the flexibility of the counter in shape with a small photo device can identify particles covering a large effective area.
\end{abstract}

Technology and Instrumentation in Particle Physics 2014,

2-6 June, 2014

Amsterdam, the Netherlands

\footnotetext{
* Speaker.
} 


\section{Introduction}

In high energy physics experiments, an aerogel Cherenkov counter (ACC) as a threshold type particle identification device has contributed to separate a kind of particle with high momentum (a few $\mathrm{GeV} / \mathrm{c}$ ). A particle identification at the trigger stage is typically used silica aerogel [U] as a radiator, which has following properties: low density, high transparency and configurable refractive index. Generally, it is difficult to design the ACC of large area, thinner type, special shape and working in magnetic field. A photo-device must be placed at a position where the detector is not irradiated directly from particles. Particularly, a photomultiplier tube cannot be used in a strong magnetic field. We have been developing a multipurpose aerogel Cherenkov counter which enables to identify charged particles at any environments [[], []].

\section{Prototype counter using WLS fibers}

The multipurpose Aerogel Cherenkov counter is composed of an aerogel radiator, a light guide made of 4 kinds of wavelength shifting (WLS) fibers and a small photo-device (PMT or SiPM) as shown in Fig. 1.
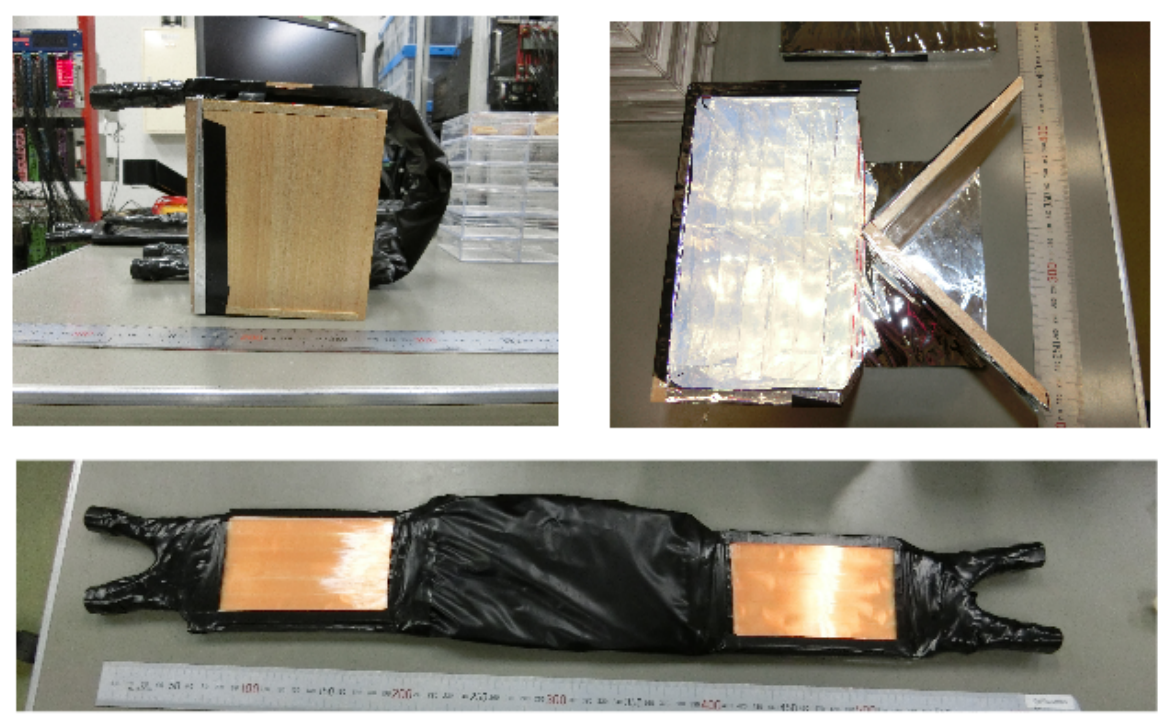

Figure 1: Prototype of multipurpose aerogel Chernekov counter. The fiber light guide is set at a space of not irradiated directly from particles and the effective area is $120 \times 100 \mathrm{~mm}^{2}$ as can be seen from the front view (top left). Cherenkov light emitted from the aerogel is reflected by a V-shaped reflector and collected into the fiber light guide as can be seen from the side view (top right). The fiber light guide has two surfaces of the effective area of $60 \times 100 \mathrm{~mm}^{2}$ (bottom).

WLS fibers made by Kuraray Co., Ltd. have the following properties: 4 kinds of absorption and emission wavelengths (B- 3, Y-11, O-2, and R-3), with a collection efficiency of 5.4\% by the double cladding, the attenuation length of about $3 \mathrm{~m}$ and $0.2 \mathrm{~mm}$ minimal diameter [䧃]. Cherenkov light emitted from the aerogel radiator is transferred to the both ends of the fiber by the total reflection condition. Since the Cherenkov light has continuous wavelength spectrum, the total collection efficiency would be improved by using 4 kinds of WLS fibers. An absorption wavelength 
region of Y-11 has range of the emission wavelength region of B-3, so the light leaked from the fibre layer would be gotten a chance to be absorbed by other fiber layer; i.e., it is expected that the efficiency would be improved more by combination of kind of WLS fiber.

Cherenkov light emitted from the aerogel is reflected by a V-shaped reflector, enters into the fiber light guide and is transferred to 4 ends of the fibers. The cross section of each end of the light guide is about $7 \mathrm{~mm}$ in diameter. The PMT used in it is R9880U-210 and R9880U-20, which have following properties: compact size $(8 \mathrm{~mm}$ diameter of photo-cathode), metal package, fast time response and high quantum efficiency [[]].

\section{Performance Measurement}

We performed measurement to evaluate the performance of the prototype counter with a test beam line at Research Center for Electron Photon Science at Tohoku University, where a positron is produced from a gamma ray with momentum of a GeV/c. Two scintillation trigger counters were put at the upstream and downstream ends of the aerogel counter. Cherenkov light emitted from the radiator enter into the fiber light guide reflected by the reflector set to 45 degrees. The efficiency and the number of detected photoelectrons were decided by an ${ }_{4} C_{1}$ combination logic circuit from 4 PMT's detection events with 0.5 p.e. threshold of ADC. There are individual differences of fiber light guide such as combination of kinds of WLS fibers, number of layers, coating the surface with an adhesive for fixing fibers and so on. Measurement items are (1) the collection efficiency of the fiber light guide, (2) the total detection efficiency, (3) the position uniformity of beam incidence and (4) the timing resolution.

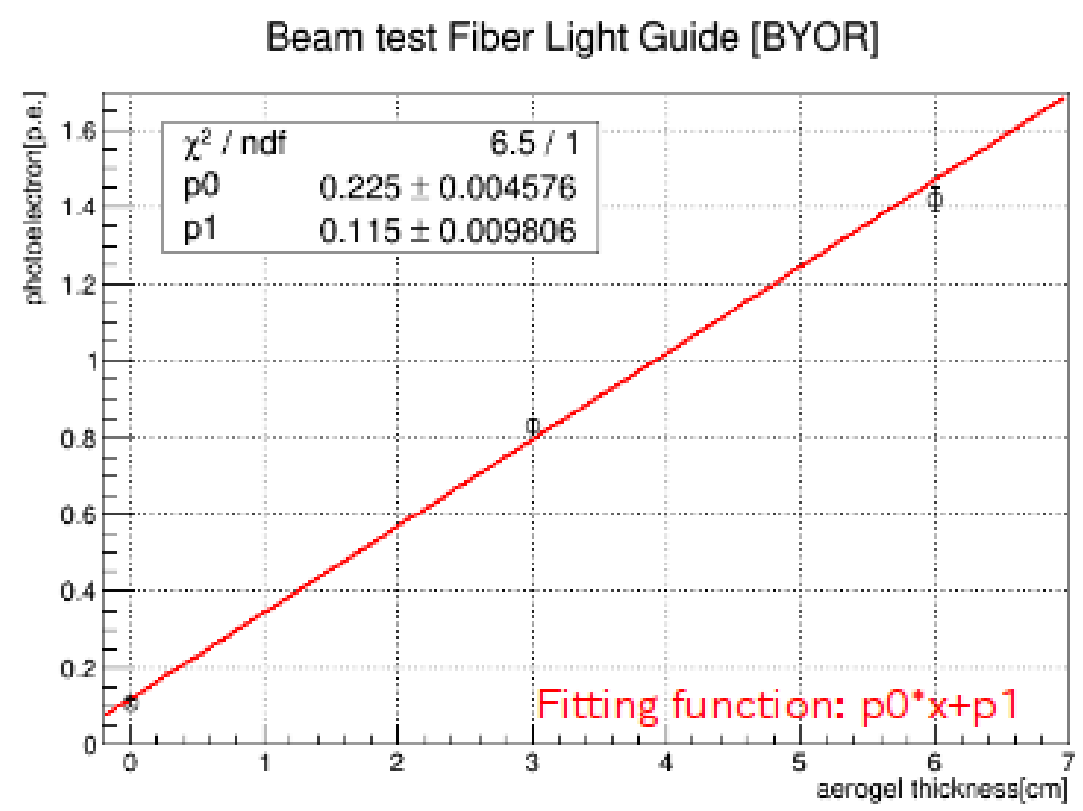

Figure 2: Since we observed an increasing number of photoelectrons in proportion to the thickness of the aerogel, reading the Cherenkov light from the light guide using a WLS fiber was succeed. 
Table 1: The result of collection efficiency of the fiber light guide

\begin{tabular}{cccc}
\hline \hline layers of the fibers & effective area[mm $\left.\mathrm{mm}^{2}\right]$ & p.e. & collection efficiency \\
\hline BYOR & $60 \times 100$ & $1.43 \pm 0.03$ & $8.1 \%$ \\
BBYY & $60 \times 100$ & $1.14 \pm 0.03$ & $6.4 \%$ \\
BY & $60 \times 100$ & $1.19 \pm 0.03$ & $6.7 \%$ \\
BBYY + coating & $60 \times 100$ & $1.42 \pm 0.03$ & $8.0 \%$ \\
BYOR & $120 \times 100$ & $1.31 \pm 0.03$ & $7.4 \%$ \\
\hline Large PMT direct & $60 \times 100$ & $17.71 \pm 0.06$ & \\
\hline \hline
\end{tabular}

The effective area of the prototype counter is $120 \mathrm{~mm} \times 100 \mathrm{~mm}$. The aerogel radiator have a refractive index of 1.05 and the total thickness of $60 \mathrm{~mm}$. Since we observed an increasing number of photoelectrons in proportion to the thickness of the aerogel, reading the Cherenkov light from the light guide using a WLS fiber was succeed (Fig. 2.). From the result shown in table 1, the collection efficiency of about $8 \%$ and the number of photoelectrons 1.43 is better in this list, where the collection efficiency is the ratio with a number of photoelectrons of large PMT directly instead of the fibers. we obserbed the timing resolution of $140 \mathrm{ps}$, a difference between the arrival times of the Cherenkov light less than 100ps at the incident position a few $\mathrm{cm}$ and a photoelectrons uniformity of $90 \%$ or more in the incident position. As compared to BBYY and BBYY + coarting in the list, an improvement of collection efficiency by making a beautiful sheet using adhesive bonds was observed.

\section{Summary}

We made a prototype of the multipurpose aerogel Cherenkov counter and performed measurement for the performance evaluation. Since we observed an increasing number of photoelectrons in proportion to the thickness of the aerogel, it was succeed to read the Cherenkov light from the light guide using a WLS fiber. The collection efficiency depends on the combination of kinds of WLS fiber and coating surface with adhesive mainly.

Furthermore, we have an idea of covering all of the side face of the radiator. This counter would contribute to an accuracy improvement by installing it in the existing detector and realization of physical experiments which has been difficult until now in the future.

\section{References}

[1] M. Tabata et al., Nucl. Instrum. Methods A 668 (2012) 64.

[2] H. Ito et al., IEEE NSS (2013) NPO1-94, DOI: 10.1109/NSSMIC.2013.6829686.

[3] M. Kubo et al., IEEE NSS (2011) NP3.M-34, DOI: 10.1109/NSSMIC.2011.6154581

[4] Kuraray Co. Ltd., Kuraray's Scintillation Materials. Product Catalog. Available: http://kuraraypsf.jp/.

[5] HAMAMTSU PHOTONICS K.K., Catalog: metal package photomultiplier tube R9880U series, Available: http: //www. hamamatsu.com/. 